(Aus dem physiologischen Institut der Universität Oxford.)

\title{
Ueber die elektromotorischen Wirkungen des wasserarmen Muskels.
}

Von

Armold Durisg (Wien).

(Mit 5 Textfiguren und 1 Abbildung als Beilage.)

Durch das liebenswürdige Entgegenkommen von Sir John Burdon Sanders on war es mir ermöglicht, in dessen Laboratorium eine Reihe von Versuchen an wasserarmen Muskeln auszuführen. Es ist mir eine Freude, für die ganz besondere Zuvorkommenheit, die mir daselbst erwiesen wurde, den herzlichsten Dank auszusprechen. Der lange gehegte Wunsch, auch über die elektrischen Frscheinungen an wasserarmen Organen etwas $\mathrm{zu}$ erfahren, fand hierdurch seine theilweise Frfüllung. Wie aus dem Folgenden hervorgehen wird, sind die betreffenden Versuche noch nicht abgeschlossen, sondern harren noch ihrer Erweiterung und eigentlichen Beendigung, die hoffentlich in Bälde an einem eigenen Instrumente möglich sein wird. Es sollen daher die nachstehenden Angaben mehr den Charakter einer vorläufigen Mittheilung als den eines abgeschlossenen Ganzen tragen.

Die Versuche sind am Capillarelektrometer unter den bekannten Bedingungen ausgeführt, wie sie von $\mathrm{Burd}$ on $\mathrm{Sanderson}{ }^{1}$ ) beschrieben wurden. Die Muskelpräparate (Sartorien) der curaresirten Frösche befanden sich in der feuchten Kammer horizontal ausgespannt und waren durch ein geringes, nahe der Achse angreifendes Gewicht belastet, so dass keine Schleuderungen auftraten. Als ableitende Elektroden dienten Baumwollfäden, die den Muskel vollkommen umfassten und dort, wo sie sich um denselben zur Schleife schlossen, durch einen dünnen Bindfaden so zusammengebunden waren, dass

1) Journal of Physiology vol. 18 p. 117. 1895. 
die Schleife weder den Muskel in der Contraction beeinträchtigte noch sich verschieben konnte. Sie führten zu den unpolarisirbaren Elektroden. Der Abstand der beiden Schlingen von einander, sowie jener, welcher zwischen ihnen und den Reizelektroden lag, wurde stets gemessen und notirt. Der letztere wurde immer so gross als nur möglich gewählt, um vor Stromschleifen in das Capillarelektrometer sicher zu sein; er schwankte um $15-20 \mathrm{~mm}$. Die Reizelektrode selbst bestand aus zwei Polpaaren, die in einer unverrückbaren Entfernung von genau $5 \mathrm{~mm}$ von einander den gemeinsamen Träger durchsetzten. Die Strecke zwischen den Polen selbst betrug fast $1 \mathrm{~mm}$, sicherte also so ziemlich gegen Stromschleifen. Da der Abstand zwischen dem peripheren und dem centralen Polpaare ein constanter war, konnten trotz der Mängel, die der Bestimmung der variablen Strecke in Folge der Messung anhaften mussten, sichere Schlüsse auf die Geschwindigkeit des Ablaufes der Erregungswelle gezogen werden. Es musste sich nämlich aus der Differenz zwischen dem Beginn der Schwankung nach centraler und peripherer Reizung, wie dies immer zwei auf einander folgenden Beobachtungen entsprach, die Fortpflanzungsgeschwindigkeit in der Zwischenstrecke ergeben. Von den 175 aufgenommenen Photogrammen entfielen 32 auf Controlversuche an normalen Thieren; der Rest vertheilte sich auf die Beobachtungen an wasserarmen Muskeln, die von Fröschen mit verschiedenem Gewichtsverlust stammten. Zur Reizung wurden immer nur einzelne Oeffnungsinductionsschläge derselben Richtung verwendet.

An Stelle der Tabellen seien funf Reproductionen von Photogrammen wiedergegeben, welche die eigenartige Form der Stromesschwankungen zeigen; in ihnen entsprechen einer Welle der Stimmgabel 0,002 Sec. Sie sind so ausgewählt, dass die verschiedene Art, in der dieselben ablaufen, zum Ausdruck kommt. Die Wiedergabe einer normalen Controlcurve konnte fuglich unterbleiben. Das in die Augen springendste Merkmal der Capillarelektrometercurven ist wohl das Auftreten ganz eigenartiger, „wellenförmiger" Stromesschwankungen, welche sich an der Stelle der normalen Curve des zweiphasischen Actionsstromes finden. Wie Fig. 1 zeigt, können dieselben von sehr verschiedener Grösse und Zahl sein. So findet sich auf dieser Platte nur eine einzige, eben noch deutlich wabrnehmbare „Welle ${ }^{4}$, welche an einem weniger scharfen Bilde wahrseheinlich vollkommen verschwunden wäre. Die Platte stammt von einem Thier mit $14 \%$ Gewichtsverlust. Viel deutlicher tritt die Erscheinung 
Ueber die elektromotorischen Wirkungen des wasserarmen Muskels. 459 bereits in Fig. 2 hervor, die von einem Frosch mit 16,7\% Gewichtsverlust herrührt. Wie sehr übrigens die Zahl der Wellen zu schwanken

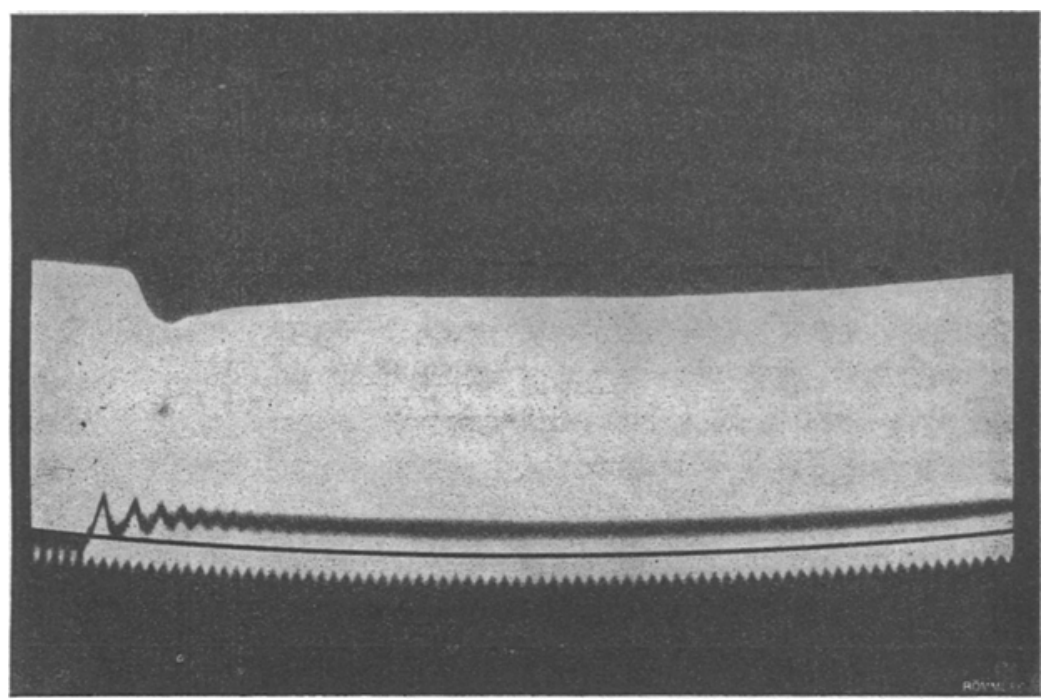

Fig. 1.

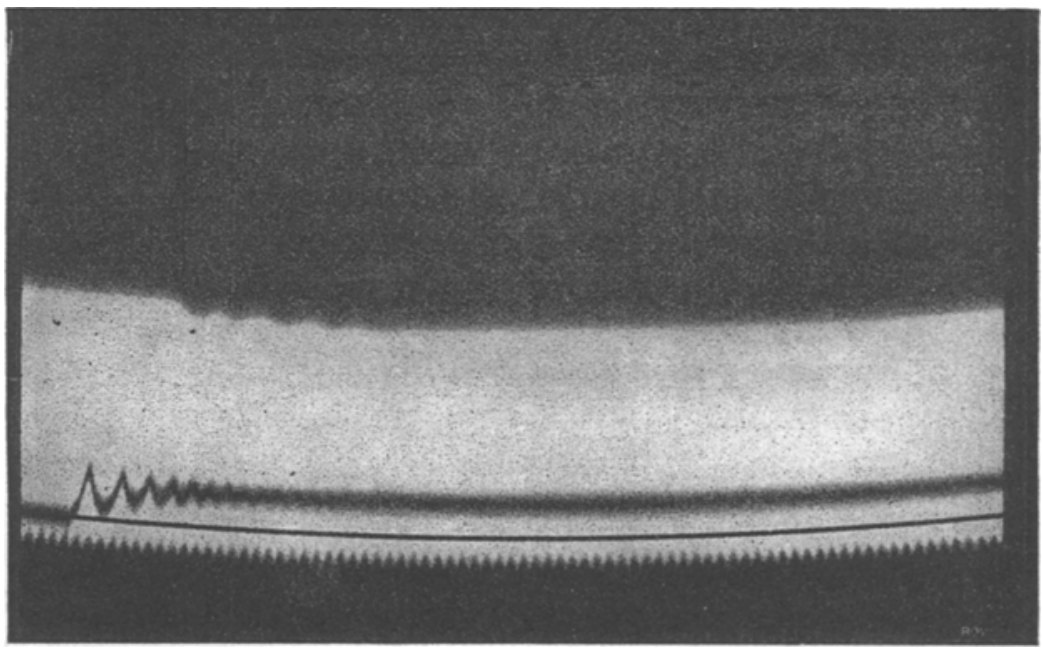

Fig. 2.

vermag, zeigen die folgenden drei Figuren, welche von Fröschen mit 17,8-18,2\% Gewichtsverlust gewonnen wurden. 
Zu ganz übereinstimmenden Ergebnissen führen auch alle übrigen Versuche, die von Fröschen stammen, deren Gewichtsverlust zwistehen

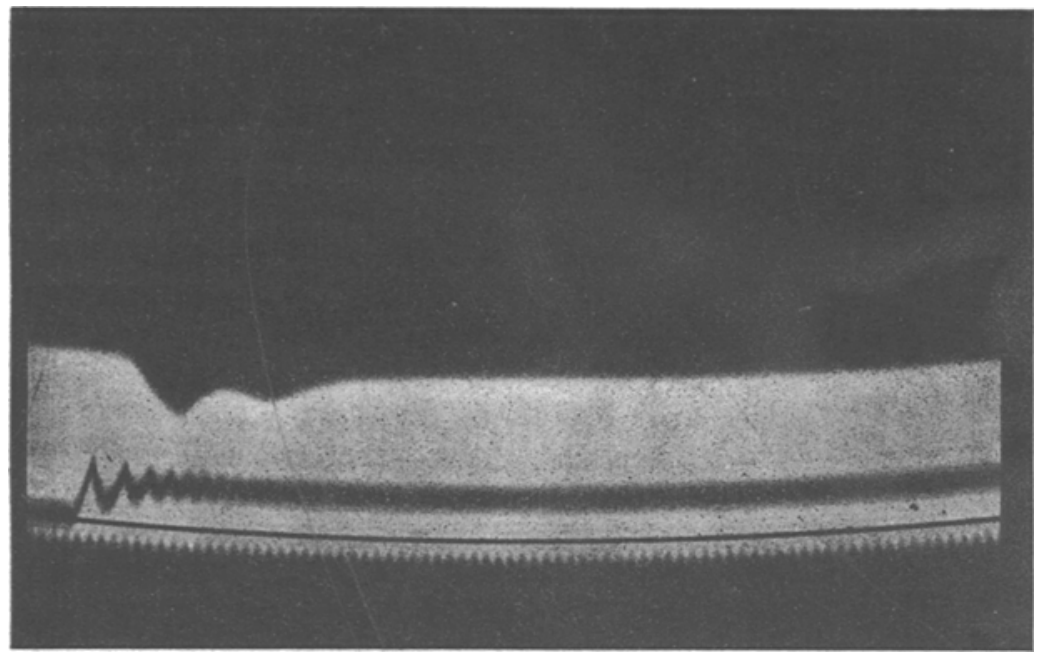

Fig. 3.

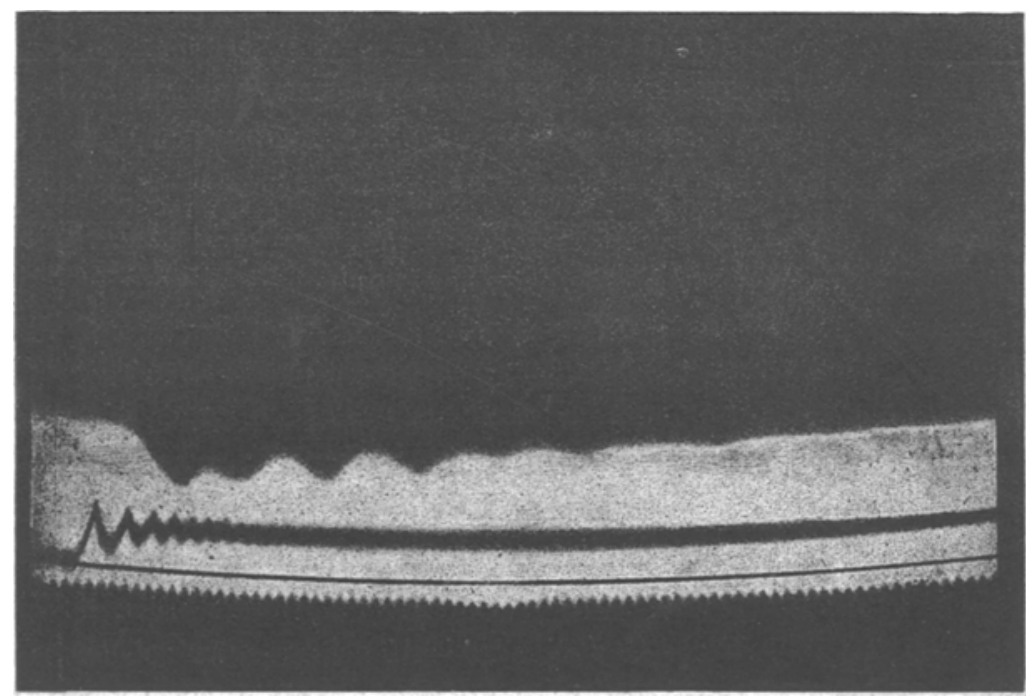

Fig. 4.

$14-20 \%$ des ursprünglichen Gewichtes betrug. In allen finden sich mehr oder minder hohe, wellenförmige Stromesschwankungen, deren 
Dauer zwischen $0,008-0,015$ Sec. liegt. Man muss dabei freilich von einigen Versuchen absehen, bei welchen die Schwankungscurve überhaupt sehr niedrig ausfiel, in diesen fehlten auch die Zeichen für das Bestehen von "Wellen"; meist bandelte es sich dabei um wenig erregbare Thiere bezw, um irgend welchen Fehler am Elektrometer. Anscheinend vermindert sich die Zahl der Wellen, welche in einem Muskel auftritt, mit der Ermüdung desselben. Ein Einfluss der Temperatur auf den Rhythmus wurde nicht geprüft. Am bemerkenswertesten erscheint es, dass nach den Versuchen nur innerhalb eines

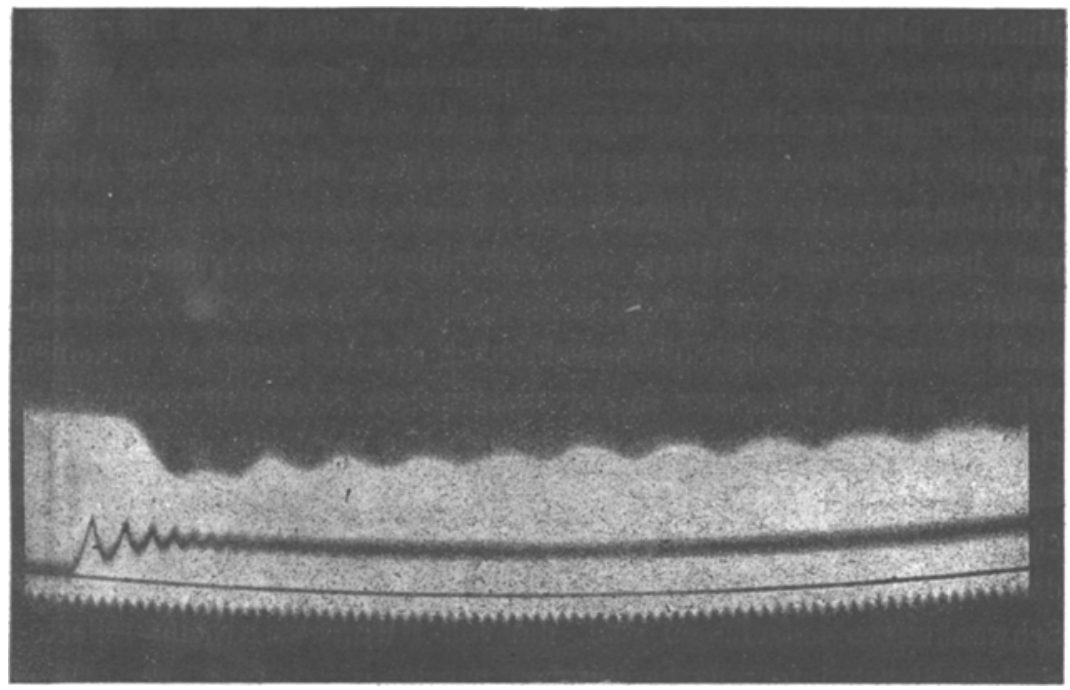

Fig. 5.

ziemlich eng begrenzten Gebietes hinsichtlich des Wasserverlustes auf einen einzigen Oeffnungsschlag rhythmische ${ }_{n}$ Wellen " folgen, denn sie verschwanden stets, wenn Frösche verwendet wurden, deren Gewicht in Folge des Durstens um mehr als $20^{\circ} \%$ abgenommen hatte, während sie auch bei den relativ beträchtlichen Wasserverlusten, wie sie einem Gewichtsverluste von $12 \%$ entsprechen, noch in vielen Fallen fehlten. Von allen Thieren, welche sich in ihrer Gewichtsabnahme ausserhalb dieser "Grenzen hielten, ergaben sich immer nur monophasische (nach Querschnittsanlegung) oder diphasische Schwankungscurven, die auf den ersten Blick von jenen eines normalen Froschmuskels nicht $\mathrm{zu}$ unterscheiden waren. Gerade diese letzte Thatsache spricht dagegen, dass es sich bei den Schwankungs- 
curven etwa um den Ausdruck einer Dauercontraction gehandelt habe, wie sie bekanntermaassen nach heftigen Reizen eintritt, da gerade an weniger wasserarmen Muskeln, die erregbarer sind als jene länger durstender Frösche, dieselbe Erscheinung hätte hervortreten müssen. Uebrigens spricht auch der Umstand allein genügend gegen eine solche Annahme, dass stets nur mit einem kleinen Inductorium, mit einem D a n i ell - Element im primären Kreis, gereizt wurde, wobei immer noch 1,7-5 cm Rollenabstand eingehalten wurden. Es dürfte die Reizstärke demnach sich wenig von jener unterschieden haben, die man im Allgemeinen für die maximale Erregung curaresirter Muskeln überhaupt verwendet. Auch der Umstand wäre hier noch zu erwähnen, dass an keinem der normalen Controlfrösche, welche unter genau denselben Bedingungen untersucht wurden, irgend eine "Welle" von jenem eigenthümlichen Charakter, wie er sich aus obigen Abbildungen ergibt, zur Beobachtung gelangte, ebenso wenig wie solche von irgend einem Autor, der mit einzelnen Inductionsschlägen arbeitete, je an einem normalen Muskel beschrieben wurden. Es besteht demnach sicherlich die Berechtigung, das Auftreten von einzelnen Wellen und Wellenzügen in der elektrischen Schwankungscurve auf einen Einzelreiz als den Ausdruck typischer rhythmischer Potentialänderungen im wasserarmen Muskel anzusehen, die für diesen charakteristisch sind. Es möge bereits hier vorweggenommen sein, dass diese Wellen als "wavelets", kleine Wellen im Sinne jener Schwankungen aufzufassen sind, die von $\mathrm{Buch}$ anan beim reflectorischen Strychnintetanus beschrieben wurden.

$\mathrm{Zu}$ erwähnen wäre hier noch, dass die oben wiedergegebenen Copien der direct erhaltenen Curven noch dem Reductionsverfahren hätten unterworfen werden müssen. Da die Versuche aber an grösseren Fröschen wiederholt werden sollen und das Wesen der Curve auch ohne die Correctur derselben deutlich erkennbar ist, konnte dies unterbleiben.

Naturgemäss denkt man bei dem Auftreten der eigenthümlichen Wellen sofort an jenen Contractionsverlauf, der in der Zuckungscurve des wasserarmen Muskels seinen Ausdruck findet. Das als Beilage angefügte Curvenblatt möge ein Bild derselben geben. Neben einer Controlcurve vom normalen Muskel 8 enthält sie Zuckungscurven von Fröschen mit verschiedenem Wasserverlust. Als Untersuchungsobject diente in diesem Falle der Musculus gracilis. Die Frösche waren so ausgewählt, dass ungefăhr bei allen Muskeln im 
Ueber die elektromotorischen Wirkungen des wasserarmen Muskels. 463

Normalzustande dieselben Bedingungen herrsehten; demnach waren nur Männchen von demselben Körpergewicht und derselben Länge verwendet worden.

Als Zeit entsprachen $1 \mathrm{~mm}$ Abscisse 0,005 Sec. Wenn der Verlauf dieser Curven im Folgendem zum Vergleich mit jenen des Sartorius herangezogen wird, so geschieht dies zu dem Zwecke, um an der Hand der etwas grösseren Curven, die der Gracilis liefert, die wesentlichsten Punkte berühren zu können. Sieht man von den vier ersten Curven der Abbildung ab, so ergibt sich aus dem Verlaufe von 5, 6 und 7 im Vergleich zur Normalcurve 8, dass der Wassergehalt des Muskels innerhalb weiter Grenzen schwanken kann, ohne dass eine nennenswerthe Aenderung des ansteigenden Curvenschenkels auf den ersten Blick erkenntlich wäre. Curve 5 rührt nämlich von einem Thier mit 12,8\% Wasser(Gewicht)verlust her, Curve 6 von einem solchen mit $14 \%$ und Curve 7 von einem Frosch, der um 19,8\% abgenommen hatte. Die Reizstärke der einzelnen Oeffnungsinductionsschläge war in allen Fällen dieselbe gewesen; es hatte sich demnach auch in der Erregbarkeit durch den gegebenen Wasserverlust kaum etwas Wesentliches geändert. Ebenso lässt sich wegen des Parallelismus zwischen den ansteigenden Schenkeln der Curven 7 und 8 ein auffallender Unterschied im Mechanismus der Contraction nicht erkennen; auch bleibt die Zeit, welche bis zum Erreichen des Verkürzungsmaximums erforderlich ist, im Grossen und Ganzen dieselbe. Die Dauer, während welcher der maximale Contractionszustand annähernd erhalten bleibt, ist ebenfalls nicht um sehr viel grösser geworden, wenn auch immerhin eine ganz merkliche Zunahme bereits erkennbar ist. Ganz anders verhält es sich aber hinsichtlich des absteigenden Curvenantheiles; dieser enthält ebenso wie bei der Ermüdung den auffallendsten Theil der Veränderung. Bereits in Curve 5 ist eine wesentliche Verlängerung desselben erkennbar, die um so grösser ausfällt, je weiter der Wassergehalt sinkt. Die dadurch bedingte Verflachung der Curve im "Stadium der sinkenden Energie" führt zum Wachsen der Curvenfläche. Nimmt man schlechtweg an, dass die längere Dauer, welche der Wiederverlängerung des wasserarmen Muskels entspricht, in directem Zusammenhang mit jenen wellenförmigen Stromesschwankungen steht, die das Capillarelektrometer anzeigt, so folgt daraus eine wichtige Consequenz für die Natur des Contractionszustandes im wasserarmen Muskel. Die graphisch ermittelte Latenz eines Muskels nämlich, wie er Fig. 5 entspricht, ist 
nach den Auseinandersetzungen in einer fruheren Mittheilung mit 0,006 Sec. $^{1}$ ) anzusetzen; es fällt demnach immer noch das Anwachsen der Negativität der erregten Stelle, das ohne Latenz einsetzt - davon soll weiter unten noch die Rede sein -, in den Theil der Contraction, der schon dem ansteigenden Curvenschenkel des Myogramms entspricht. Das Maximum der Negativität ist aber erreicht, bevor die maximale Verkürzung des Muskels graphisch zum Ausdruck gekommen ist. Es ergibt sich dies wenigstens, wenn man die Zuckungscurve eines gleich stark wasserarmen Sartorius mit der obigen Schwankungscurve vergleicht. Nun folgt aber die Rückbewegung des Meniscus im Elektrometer im Sinne einer Abnahme der Negativität, während nach den zeitlichen Verhältnissen die Zuckungshöhe noch zunimmt. Etwa 12-14 $\sigma$ nach dem Reizmoment ist die erste grosse Schwankungswelle abgelaufen und wieder ein Minimum an Negativität, das ist ein Maximum an Positivität, erreicht; während dessen steigt aber die Curve der Zuckung immer noch an, so dass anzunehmen ist, dass die beobachteten Wellen mit dem Arfangstheile, dem Gipfel der Zuckungscurve und eventuell noch den ersten Momenten der beginnenden Wiederverlängerung zusammenfallen. Dagegen kann als sicher angenommen werden, dass dem gleichzeitig mit der Stromesschwankung graphisch aufgezeichneten Stadium der wesentlich verzögerten Wiederverlängerung bereits kein Vorhandensein von nachweisbaren "Wellen" mehr entspricht. Nun findet sich aber am normalen Muskel, bei fast derselben Geschwindigkeit der Energiezunahme, im Elektrometerphotogramm ausser jener ersten grossen Welle - der negativen Schwankung des Demarcationsstromes oder dem zweiphasischen Actionsstrom - keine weitere Stromschwankung; es muss daher naheliegend erscheinen, doch die beiden wesentlichen Aenderungen gegenüber der Norm $\mathrm{zu}$ einander in Beziehungen zu bringen und den Ablauf der Erregungswellen mit der länger dauernden Contraction und verzögerten Wiederverlängerung nach dem Erreichen des Verkürzungsmaximums zusammenzustellen.

Es würde aber dann der elektrische Effect dem mechanischen voraneilen. Dies darf aber nicht befremden, denn nach allen Resultaten, die man bei graphischen Studien uber die zeitliche Folge von Vorgängen im Muskel erbält, ist es sicher, dass wir erst relativ beträchtliche Zeit später von denselben unterrichtet werden, da diese Vorgänge eine gewisse Zeit gedauert haben müssen und

1) Dieses Archiv Bd. 87. 
Ueber die elektromotorischen Wirkungen des wasserarmen Muskels. 465

auch ein bestimmtes Ausmaass an Grösse aufweisen müssen, damit sie der graphischen Registrirung zugänglich sind; dem wasserarmen Muskel scheint die Möglichkeit der Zunahme dieser „Latenz der Methode" aus später anzuführenden Gründen nur noch naheliegender, so dass das Voranlaufen der Erregungswelle vor der Contractionswelle nur noch ausgesprochener werden muss. Da nach Lee $^{1}$ ) übrigens eine Uebereinstimmung zwischen der Dauer der Zuckung und jener der Stromschwankungen wahrscheinlich ist, so ist im Zusammenhalt mit dem Umstande, dass die "Wellen" den Zeitpunkt, in dem die maximale Verkürzung erreicht ist, überdauern können, auch die oben ausgesprochene Vermuthung gestützt, dass die langsame Wiederverlängerung des Muskels nicht allein durch eine rein mechanische Behinderung derselben bedingt ist, sondern dass wirklich eine Beziehung zwischen der längeren Dauer der Verkürzung und der wellenförmigen Stromschwankung besteht. Es ist demnach anzunehmen, dass ausser der klebrig-teigigen Beschaffenheit des wasserarmen Muskels, die einer rein mechanischen Verschiebung der Theilchen einen gewissen grösseren Widerstand entgegensetzen muss als im normalen Muskel, noch ein Fortdauern des Erregungszustandes stattfindet, das sich wenigstens auf der Höhe der maximalen Contraction noch im rhythmischen Wechsel zweier entgegengesetzt verlaufender Processe kennzeichnet.

Die bisher besprochenen Curven bezogen sich nur, auf gewisse mittlere Wasserverluste, bei denen eben die eigenthümlichen Erscheinungen der "Wellenbildung" auftraten. Es muss daher noch jener Versuche gedacht werden, in denen die Wasserverluste grössere oder geringere waren und rhythmische Schwankungen fehlten. Wenn bei einer Gewichtsabnahme der Thiere unter $12 \%$ keine so bedeutenden Aenderungen der phasischen Actionsströme zu bemerken waren, dass ein Unterschied gegen die Norm zu erkennen gewesen wäre, so vermag dies kaum auffallend zu erscheinen, nachdem auch an der Zuckungscurve nur mehr geringe Abweichungen wahrnehmbar sind. Einer Erklärung wird es daher nach dieser Richtung nicht bedürfen, nachdem bereits bei Wasserverlusten von 14 und $13 \%$ die Höhe und Zahl der Wellen schon abgenommen hatte, für deren Auftreten das Optimum bei etwa 16-18\% Gewichtsverlust lag; dagegen wird die Frage, warum bei einer Gewichtsabnahme von

1) Archiv für (Anat. und) Physiologie 1887 S. 210. 
mehr als $20 \%$ keine „Wellen" aufgetreten seien, um so mehr eine Antwort fordern. Zwei Beispiele, die von einem entsprechenden Thiere stammen, gibt das Curvenblatt unter 3 und 4. Es handelt sich um einen Wasserverlust von $26,2 \%$; dabei ist Curve 4 bei derselben Reizstärke gewonnen wie die folgenden, bereits besprochenen Zuckungscurven. Die Zuckung fiel ungleich niederer aus und zeigt einen ganz gewaltigen Verkürzungsrückstand, der nur sehr langsam zurückgeht. Die mit $6 \mathrm{~cm}$ geringerem Rollenabstand aufgenommene Curve 3 gibt das nämliche Bild nur vergrössert wieder; der Verkürzungsrückstand beträgt hier am Ende der eigentlichen Zuckung noch die Hälfte der gesammten Hubhöhe. Aber auch das Stadium der steigenden Energie hat sich wesentlich verlängert, indem es um mehr als die Hälfte gegen die Norm zugenommen hat; dabei ist die Zuckungshöhe trotz der stärkeren Reizung noch weit unter jener des normalen Muskels zurückgeblieben. Gerade die beiden letzteren Momente sprechen dafür, dass die Scbädigungen hier schon viel weitergehende gewesen sind als bei den mittleren Wasserverlusten. Auch das Elektrometer gibt eine Antwort in demselben Sinne. Man erhält ungemein flach verlaufende Schwankungscurven, an denen die zweite Phase so nieder ist, dass eine Abgrenzung derselben nicht möglich wird. Man vermag an denselben auch den Moment des Beginnes der ersten Phase nur höchst ungenau zu ermitteln, da der Anstieg auch weniger steil beginnt, wie dies der Zuckungscurve ganz gut entspricht. Dass hierbei keine Wellen mehr wahrgenommen werden konnten, scheint desshalb ganz erklärlich. Man muss übrigens auch bedenken, dass das Auftreten dieser wie überhaupt jener aller Schwankungsformen an und für sich beeinträchtigt sein musste, da es sich um matte, träge Thiere handelte. Wie bekannt, erhält man selbst von anscheinend ganz normalen Fröschen nur niederere Elektrometercurven, wenn sich jene unter weniger günstigen Bedingungen längere Zeit in Gefangenschaft befanden. In ganz demselben Sinne sprechen auch die Beobachtungen von Engelm an $n^{1}$ ), nach denen an der Haut die elektromotorische Wirksamkeit mit dem Sinken des Wassergehaltes ganz wesentlich abnimmt, und zwar nicht allein deswegen, weil dadurch der Widerstand ein grösserer geworden ist, sondern weil eine directe Verminderung der elektromotorischen Eigenschaften zu Stande kommt. Ganz das Nämliche gilt ja auch

1) Dieses Archiv Bd. 4 S. 146. 
Ueber die elektromotorischen Wirkungen des wasserarmen Muskels. 467

für die Ströme, welche an der Froschzunge zur Beobachtung gelangen. $\mathrm{Ob}$ es daher möglich sein wird, mit einem empfindlicheren Capillarelektrometer doch noch Wellen nachzuweisen, muss für den sehr wasserarmen Muskel als recht fraglich bezeichnet werden. Gelingt dieser Nachweis mit diesem Instrumente nicht, so ist auch vom Galvanometer einerseits wegen der zu grossen Trägheit ein Resultat nicht zu erwarten, andererseits schliesst sich ein Rheotomverfahren für den wasserarmen Muskel aus. Dieser ist nämlich stets nur für ganz wenige Einzelzuckungen verwendbar, da er sehr rasch ermüdet und auch ständig die Abgleichung der Verkürzungsrückstände abgewartet werden muss, denen man nur mit ganz geringen Gewichten entgegenarbeiten darf. Die Unmöglichkeit, vorläufig über stark wasserarme Muskeln sichere Angaben zu machen, war der Grund, warum im Bisherigen, aber auch im Folgenden nur von den Beobachtungen bei mittelgrossen Wasserverlusten die Rede sein soll.

Der rhythmische Wechsel zwischen örtlicher Ruhe und Erregung, dessen Ausdruck wir in der wellenförmigen Schwankungscurve finden, liefert eine weitere interessante Beziehung zu anderen Versuchen, die wir Kühne ${ }^{1}$ ) und Biedermann ${ }^{2}$ ) verdanken. Biedermann beschreibt, dass die Muskeln todter und enthäuteter Frösche, die frei an der Luft liegend der Austrocknung überlassen werden, eine hochgradige Erregbarkeitssteigerung aufweisen, ausserdem aber die Eigenschaft besitzen, auf streng localisirte Reize mit kräftiger, langanhaltender Dauerverkürzung zu antworten, die nicht nur anf den direct erregten Muskel beschränkt bleibt, sondern sich in weitem Umkreise anderen Muskeln mittheilt. Ganz in derselben Weise ver-

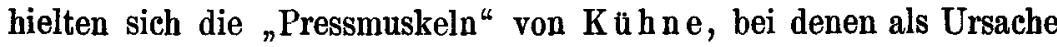
der Miterregung benachbarter Fasern nach Biedermann ${ }^{3}$ ) ebenfalls die Verarmung an Wasser anzusehen ist. Auch Langendorff ${ }^{4}$ ) beschrieb ähnliche Beobachtungen, die er an Fröschen machte, denen Glycerin unter die Haut injicirt war. Biedermann glaubt nun, dass nicht die Erregbarkeitssteigerung die Ursache für die Miterregung anderer Fasern und Muskeln sei, da eine solche von Muskeln aus, die mit $\mathrm{Na}_{2} \mathrm{CO}_{3}$ behandelt sind, nicht stattfindet, eben-

1) Zeitschrift für Biologie Bd. 24 u. 26.

2) Sitzungsber. der Wiener Akademie der Wissensch. Bd. 97 H. 3.

3) Elektrophysiologie. Fischer, Jena 1895. S. $363 \mathrm{u}$. ff.

4) Arch. f. (Anat. u.) Physiologie 1891 S. 480. 
sowenig wie anzunehmen ist, dass die Form der Zuckungscurve des primär erregten Muskels der Grund für dieselbe sein kann, denn auch andere Dauerregungen, wie z. B. am $\mathrm{NH}_{3^{-}}$oder Veratrinmuskel, geben $\mathrm{zu}$ keiner Miterregung Anlass. Die Ursache für diese liegt demnach nach der Angabe des Autors in einer Erregung benachbarter Muskelfasern durch das Auftreten der Actionsströme im primär gereizten Muskel.

Wie bereits oben ausgeführt, bestehen zwischen dem postmortal trocknenden Muskel und demjenigen, der erst im Laufe von 1 bis 11/2 Tagen bis zu den angegebenen Grössen an Wasser verlor, hinsichtlich der Erregbarkeit Unterschiede, indem bei diesen letzteren eine Erregbarkeitssteigerung nicht zu Stande kommt, ja, im Gegentheil eine Verminderung der Anspruchsfähigkeit auf Oe.-S. beobachtet wird. Dementsprechend kann man den enthäuteten Schenkel eines durstenden Frosches, auch wenn er $30 \%$ an Gewicht verloren hatte, beliebig mechanisch reizen, ohne mehr als eine örtliche, träge Contraction auszulösen; schwächere Reize bleiben überhaupt erfolglos; führt man dasselbe nur eine Viertelstunde später am frei an der Luft liegenden Präparate aus, so bemerkt man sofort das von Biedermann beschriebene Verhalten. Es zeigt dies also, dass beide Arten wasserarmer Muskeln nicht ohne Weiteres identificirt werden dürfen. Tödtet man einen normalen Frosch und lässt ihn ebenso wie Biedermann liegen, so beobachtet man z. B. nach acht Stunden die Erscheinung in ganz auffallender Weise. Eine Erschütterung der Glasplatte genügt, eventuell schon tetanische Zuckungen auszulösen; eine leise Berührung mit der Spitze der Pincette führt zu tetanischer, fast coordinirter Contraction der nahe gelegenen Muskelgruppen. Präparirt man nun einen der Muskeln, von dem aus es gut gelang, die Miterregung benachbarter Muskeln auszulösen, vorsichtig heraus, wobei natürlich zuerst tetanusartige Contraction auftritt, und gibt ihn in das Myographion, so erbält man dann, wenn man nach einiger Zeit reizt, den Muskel aber vor weiterer Vertrocknung geschützt hat, eine Muskelcurve, die gar nichts von einem tetanischen Charakter mehr an sich hat, sondern ganz genau der eines gewöhnlichen wasserarmen Muskels entspricht; das Curvenblatt gibt unter Curve 2 eine solche wieder. Entfernt man aber nun die schützenden Muskelstücke, die mit ihren wasserarmen Aussenseiten dem untersuchten Präparat anlagen, während sich dies erholen konnte, und lässt nun das Präparat - in diesem Falle war es ein Gracilis - 
an seiner Oberfläche weiter eintrocknen und reizt nun, so erhält man eine vollkommen tetanische Curve, wie sie als erste auf Fig. 6 gezeichnet ist. Es ist wohl zweifellos, dass ein Elektrometerphotogramm von dieser Zuckung durch eine ganze Secunde auch tetanische Actionsströme gezeigt hätte, die sich als grosse Wellen abgebildet hahen müssten, da auch die Hubhöhe gegenüber Curve 2 um fast das Doppelte gestiegen ist. Da die Zuckungscurve des postmortal trocknenden Muskels somit wesentliche Unterschiede aufweisen kann, wäre es gewiss nicht unlohnend zu untersuchen, ob bei einer Stromesschwankung, die dem Verlaufe von Curve 1 entspricht, das erste Auftreten von Wellen bereits in den ansteigenden Curvenschenkel fällt, was nach den Erfahrungen am Muskel, der in vivo dem Wasserverlust unterworfen war, zweifellos ist. Zwischen Curve 1 und 2 würde ein Unterschied im Elektrometerphotogramm ausser groben Schwankungen nur in der Höhe der Wellen und der Dauer, während deren sie auftraten, bemerkbar gewesen sein. Wenn Biedermann anführt, dass die Reizung tiefer gelegener Schichten nicht so sicher zur Auslösung eines secundären Tetanus von Muskel zu Muskel führt, so steht dies mit dem Fehlen der Wellen an Muskeln von geringem Wasserverlust wohl in ganz guter Uebereinstimmung, da es einem Zweifel nicht unterliegt, dass gerade die oberflächlichen Schichten postmortal an Wasser verlieren, während tiefer gelegene Theile von mehr normalem Wassergehalt zu sein scheinen, von diesen somit eine Wirkung im Sinne der Biedermann'schen Erscheinung nicht zu erwarten ist. Diese selbst muss aber nach zwei verschiedenen Richtungen hin betrachtet werden. Einerseits handelt es sich um das Auftreten specifisch tetanischer Erscheinungen, die nur dann zu Stande kommen, wenn die Oberfläche des Muskels einen gewissen Grad von Trockenheit erlangt hat. Andererseits um eine Verlängerung des Stadiums der steigenden und sinkenden Energie, ohne den Charakter eines Tetanus, jedoch mit lange anhaltendem Verkürzungsrückstand; beiden werden jedoch rhythmische Stromesschwankungen zu Grunde liegen.

Die Erklärung für das Verhalten des secundär erregten Muskels scheint dadurch ganz eindeutig gegeben, dass kräftige rhythmische Schwankungen im primär erregten Muskel zu secundärem Tetanus der anliegenden Muskeln Anlass geben, während die geringen Höhen, welche die jeweiligen Potentialdifferenzen am Muskel mit geringerem, mehr gleichmässigem Wasserverlust erreichen, entweder nur eine 
relativ kurzdauernde Zuckung der benachbarten Muskeln zur Folge haben oder eine solche überhaupt nicht mehr auszulösen vermögen, je nachdem der Zuckungsverlauf des primär erregten Muskels ein tetanischer oder bloss verflachter und verzögerter war. Wegen des Fehlens rhythmischer Schwankungen bei anderen Formen der Dauercontraction, so z. B. bei Veratrin $\left.\left.{ }^{1}\right)^{2}\right)^{3}$ ), ist das Wesen der secundären Erregung von Muskel zu Muskel also wohl im Auftreten der grossen rhythmischen Stromschwankungen zu suchen, wobei der von B i ed er$\operatorname{man} n$ angenommene leichtere Uebergang derselben von einer Faser zur nächsten als unterstützendes Moment herauszuziehen ist. $\mathrm{Ob}$ dieses leichtere Zustandekommen nur in einem Näherrücken der Fasern bedingt ist, somit in einer Widerstandsherabsetzung, erscheint bei der geringeren Anspruchsfähigkeit des wasserarmen Muskels auf elektrische Reize fraglich, um so mehr, als ein Muskel, von dem aus zuerst die Auslösung des Tetanus gelang, nach dem Herauspräpariren denselben nicht mehr zeigt, wohl aber nach neuerlicher weiterer Eiutrocknung der Oberfläche durch einen einzelnen Oeffnungssehlag abermals zum Tetanus veranlasst wird. Da die Ermüdung als die Ursache dieses merkwürdigen Versehwindens nicht aufgefasst werden kann, so ist es wohl eher wahrscheinlich, an eine mehr gleichmässige Vertheilung des Wassergehaltes nach der Präparation zu denken, die dann zum Auftreten der gewöhnlichen Zuckungscurve des wasserarmen Muskels führt und erst dann, wenn neuerlich durch die Oberflächenveränderung das Auftreten höherer Potentialdifferenzen, entsprechend tiefergreifenden Dissimilationsprocessen, möglich ist, zu rhythmisch verlaufenden grösseren Dissimilations- und Assimilationsvorgängen in den tiefer liegenden, noch weniger geschädigten Theilen des Muskels Anlass gibt.

Betrachtet man nun die Dauer der Wellen, welche am wasserarmen Muskel zu Stande kommen, so begegnet man abermals einem auffallenden Befunde. Die Zeit für den vollen Ablauf einer solchen schwankt nämlich, ganz gleichgültig, ob diese nun eine grössere oder kleinere war, zwischen 8,0 und $15,0 \sigma$, und diese steht in vollkommener Uebereinstimmung mit jenen Grössen, welche unter ganz anderen Bedingungen von anderen Autoren gefunden wurden. In

1) Schenk, dieses Arch. Bd. 61 u. Bd. 63 .

2) Buchanan, Journal of Physiol. t. 27.

3) Garten, dieses Archiv Bd. 77. 
seiner Abhandlung über die elektrischen Effecte nach Reizung des Muskels und ihre Beziehung zu der Zuckungscurve weist Burdon Sanderson ${ }^{1}$ ) darauf hin, dass dem Muskel bei bestimmten Formen der Dauercontraction auch ein ganz bestimmter Schwankungsrhythmus eigen sei. Aus seinen Angaben wie den Abbildungen (Taf. II Fig. 6 und Fig. 7, sowie Taf. III Fig. 1) geht hervor, dass die Dauer der einzelnen kleinen Schwankungen $8-12 \sigma$ beträgt, ein Resultat, das durch Fl. Buch an a ${ }^{2}$ ) bestätigt und erweitert wurde. Auch dieser Autor findet nämlich ganz ähnliche Zeiten für die einzelne Welle, was aus den Worten hervorgeht: ${ }_{n}$ shows a periodical response in which undulations recur at irregular intervals with a frequency of about 80 per second " 3 ), was also wieder einer Schwankungsdauer von circa $12 \sigma$ entsprechen würde; freilich fanden sich auch unter bestimmten Einflüssen etwas wechselnde Werthe, diese fallen aber doch, wie Tab. $\mathrm{H}$ ergibt, im Wesen immer in dieselbe Grössenordnung, der ein Mittel von dem genannten Werthe zukommt. Dasselbe wie am Ritter'schen Tetanus, dem die letzten Angaben galten, ergibt sich auch für den Strychninkrampf; nur sind hierbei nach Buchanan und Burdon Sanderson zwei verschiedene Typen von Rhythmen zu berücksichtigen; der eine derselben ist langsamer und entspricht 4-12 "grossen" Wellen per Secunde, denen als feine Zähnelung Schwankungen von derselben Dauer wie bejm Ritter'schen Tetanus, also etwa 50-100 "kleine Wellen ${ }^{4}$ per Secunde, aufsitzen.

Zur selben Zeit wie Buchanan beschäftigte sich auch Garte ${ }^{4}$ ) mit den "rhythmischen elektrischen Vorgängen im quergestreiften Skelettmuskel". Abermals erscheint für diese dieselbe Zeitdauer von rund 0,01 Secunde, die je nach dem Zustande des Muskels nnd der Temperatur etwas länger oder kürzer werden kann. Die Versuche beziehen sich sowohl auf jene wellenförmigen Stromesschwankungen, welche sofort nach der Anlegung eines künstlichen Querschnittes auftreten, als auch auf die elektrischen Erscheinungen beim Schliessungstetanus u. s. w. Ganz so wie $\mathrm{B}$ u ch a $\mathrm{n}$ an findet auch Garten, dass die Wellen in derselben Weise und mit dem näm-

1) Journal of Physiology vol. 18.

2) Journal of Physiology vol. 27.

3) Ibid. S. 143.

4) Abhandl. der math.-physik. Classe d. k. sächs. Ges. der Wissensch. Bd. 26. 1901. 
lichen Rhythmus ablaufen, ob das Präparat nun direct oder vom Nerven aus erregt war. Wenn nun der wasserarme Muskel, wie aus den oben wiedergegebenen Photogrammen hervorgeht, trotz der ganz wesentlichen Veränderungen, die ein Wasserverlust etwa im Betrage von einem Sechstel des ursprünglichen Gehaltes bedingt, immer noch denselben Schwankungsrhythmus zeigt, so ist dies wohl eine sehr auffallende Erscheinung, die neuerdings an die Möglichkeit des Bestehens eines bestimmten Organrhythmus denken lässt, auf den bereits Burdon Sanderson hingedeutet hat. Bei dem Hinweis auf diese Angabe $S$ anderson's erwähnt übrigens Garten ${ }^{1}$ ), dass er auch an der Curve des elektrischen Schlages von Torpedo ebenfalls Einzelsch vankungen in der Dauer von 8-12 $\sigma$ erkennen konnte, was mit den Mittheilungen von Gotch und Burch über die Entladung des elektrisehen Organes von Malapterurus in guter Uebereinstimmung steht. Bedenkt man, dass sowohl der Muskel an und für sich als auch dann, wenn er curaresirt ist, oder wenn seine Zuckungscurve durch Wasserverlust ganz wesentliche Veränderungen erfahren hat, unter geeigneten Verhältnissen dieselben wellenförmigen Schwankungen auftreten lässt, welche bei einzelnen Formen der Dauercontraction im reflectorischen Tetanus oder bei Erregung vom Nerven aus unter bestimmten Bedingungen erscheinen, die den nämlichen Rhythmus mit der Entladung elektrischer Organe zeigen, so ist die Vermuthung, dass in gewissen Grenzen ein eigener Organrhythmus besteht, wirklich keine so unwahrscheinliche.

Die theoretische Deutung des Auftretens der Erscheinung am wasserarmen Muskel stösst auf einige Schwierigkeiten. Kann man bei der Anlegung eines künstlichen Quersehnittes oder bei der Dauerdurchströmung annehmen, dass ein länger anhaltender Reiz von einer bestimmten Stelle ausgeht oder etwa eine Folge von Reizen eintrifft, wie beim reflectorischen Tetanus, so kann bei der Erregung des wasserarmen Muskels auf einen einzelnen Oeffnungsschlag nur in dem Sinne an eine längere Dauer örtlicher Erregung gedacht werden, dass an der gereizten Stelle ein ausgedehnterer Zerfall von Molekülen stattfindet. Auf die Möglichkeit einer solchen gesteigerten Hinfälligkeit wurde schon oben hingewiesen; sie vermag aber auch zu einer befriedigenden Erklärung kaum zu verhelfen, selbst wenn man annimmt, dass in Folge derselben auf einen Einzelreiz am

1) 1. c. S. 396 . 
wasserarmen Muskel eine stärkere Negativität zu Stande kommt. Denn einerseits müsste aus dem leichteren Zusammenbrechen von Molekülcomplexen fast nothgedrungen auch eine Steigerung der Erregbarkeit folgen, an deren Stelle aber gerade eine Verminderung derselben beobachtet wird, andererseits wäre es dann unerklärlich, wieso der Rhythmus derselbe bleiben konnte. Denn wenn die örtliche Negativität fortschreitend von Dissimilationsprocessen im Muskel begleitet ist, die bis zu einer bestimmten Höhe ansteigen, um dann einem Assimilationsvorgang zu weichen, der so lange anhält, bis die Erregbarkeit des Muskels für den fortdauernden Reiz zu jenem Punkte gelangt ist, an dem dieser neuerdings einen Dissimilationsvorgang hervorruft, so müsste bei dem nämlichen Rhythmus im wasserarmen Muskel auch der Assimilationsprocess mit derselben Schnelligkeit vor sich gehen wie am normalen Muskel; es dürfte aber der Dissimilationsvor'gang dann nicht einmal ein weitergehender sein als in diesem. Die Ursache des Rhythmus ist daher wohl in einer etwas anderen Form der Deutung zu suchen, die derart sein muss, dass sie ebenso gut für den Entladerhythmus der Ganglienzelle eine Erklärung gibt wie für die Erscheinungen an Nerv und Muskel.

Vergleicht man in den einzelnen Curven, welche unter denselben Bedingungen gewonnen wurden, die Dauer der gesammten Schwankung, so ergibt sich für den wasserarmen Muskel im Allgemeinen eine Verlängerung derselben, die aber freilich wegen des flachen Verlaufes im letzten Antheile in vielen Fällen mehr mit Wahrscheinlichkeit als mit unbedingter Sicherheit zu behaupten ist. Wie bekannt, besteht dadurch eine gewisse Beziehung zur Ermüdung des Muskels. Ueberraschender sind die Ergebnisse über die Feststellung des Beginnes der Schwankung und die Geschwindigkeit, mit der sich diese fortgepflanzt hat.

Aus einer früheren Mittheilung ${ }^{1}$ ) ging hervor, dass die graphisch ermittelte Latenz des Muskels mit dem Sinken des Wassergehaltes eine ganz wesentliche Verlängerung erfahren kann, die bei grossen Wasserverlusten das Vierfache jener erreicht, welche bei denselben Versuchsbedingungen am normalen Thier beobachtet wurde. Ganz abweichend davon liess sich für die Leitungsgeschwindigkeit ${ }^{2}$ ) im wasserarmen

1) Dieses Archiv Bd. 87.

2) Dieses Archiv Bd. 92. 
Nerven nur eine recht unbedeutende Zunahme erkennen, während die sogenannte Endorganlatenz eine recht grosse war. Es schien daher sehr wissenswerth, $\mathrm{zu}$ erfahren, ob auch die elektrische Zustandsänderung ebenso wie die mechanische eine Verzögerung ihres Eintrittes erfähıt; sollte dies der Fall sein, so würden sich auch für die Ermittlung, ob eine Latenz für das Negativwerden der erregten Stelle stattfindet, günstigere Verhältnisse ergeben haben. Ueber die Zeit, nach welcher in normalen Muskel die negative Schwankung des Demarcationsstromes bezw. der Beginn der ersten Phase des Actionsstromes zu Stande kommt, bestehen heute wohl kaum mehr Zweifel, nachdem es sicher ist, dass die gereizte Stelle, wie es die Theorie Hermann's fordert, mit dem Momente der Erregung negativ werden muss. Die Annahme Bernstein's ${ }^{1}$ ), dass jedes Muskelelement erst den Process der negativen Schwankung durchgemacht haben müsse, bevor es aus dem Zustand der Ruhe in den thätigen übergeht, wesshalb mit dem Eintritt des Contractionszustandes im Element auch keine Aenderung des elektromotorischen Verhaltens mehr nachzuweisen ist, ist heute wohl nicht mehr zu stützen; im Gegentheil muss das Bestreben, den Contractionsvorgang in directen Zusammenhang mit den elektromotorischen Erscheinungen des thätigen Muskels zu bringen, immer mehr zur Annahme führen, dass die Stromesschwankung entweder eine Begleiterscheinung oder vielmehr ein integrirender Theil des Processes der Muskelverkürzung ist. Demnach wird die Zeit, um welche die Actionsströme der Contraction voraneilen, eine ständig geringere, denn die Dauer der Latenz der mechanischen Zustandsänderung schrumpft immer mehr zusammen (nach Burdon Sanderson und Verfasser wenig über 0,002 Sec.), und die Dauer der nachweisbaren Potentialänderung nimmt zu (Lee). Dabei ist noch zu bedenken, dass die Latenz des Muskelelementes in allen angeführten Versuchen noch als ungleich kürzer angenommen werden muss, so dass man heute schon als erwiesen ansehen kann, dass diese höchstens noch an die Grenzen jener Zeitgrössen reicht, die eben graphisch noch ermittelt werden können. Es sagt demnach auch Burdon Sanderson ${ }^{2}$ ) bereits, dass der elektrische Vorgang, anstatt dem mechanischen voranzueilen, mit ihm fast gleichzeitig stattfinden müsse; "es muss daher jede Theorie des Erregungsprocesses im Muskel fallen gelassen werden, welche von der Voraus-

1) Siehe auch Jensen, dieses Arch. Bd. 77 S. 107.

2) Physiolog. Centralbl. 1890 S. 185. 
Ueber die elektromotorischen Wirkungen des wasserarmen Muskels. 475

setzung ausgeht, dass die elektrische Schwankung in die mechanische Latenzzeit fällt." An diesem Orte möge eine Richtigstellung einer Angabe mit den eigenen, mir brieflich mitgetheilten Worten von Sir J. Burdon Sanderson eingefügt sein, welche eine sinnstörende Wendung in derselben Mittheilung ${ }^{1}$ ) corrigirt, da der Inhalt derselben auch in andere Arbeiten, so in das Buch von Biedermann, Eingang gefunden hat.

"In meiner Mittheilung betitelt "Photographische Darstellung der mechanischen und elektrischen Veränderungen, die während der sogenannten Latenzzeit im Muskel stattfinden", wurde angeführt, dass, wenn man einen Muskel elektrisch reizt und den ersten Beginn seiner mechanischen Zustandsänderung photographisch registrirt, „das Intervall zwischen der Reizung und dem ersten Anzeichen einer Formveränderung 0,0025 Sec. hetrage". Es wurde daselbst weiter mitgetheilt, dass in einem Muskel (Gastrocnemius, der von Elektroden aus gereizt wird, die in einem Abstande von $12 \mathrm{~mm}$ dem Nerven anlagen) das Intervall zwischen Erregung und Schwankung 0,0035 Sec. betrug. Von daher rührt nun das Missverständ̉niss, dass die elektrische Schwankung der plötzlichen Aenderung der elastischen Eigenschaften des Muskels nicht vorausgeht. Die Beobachtungen sind aber dann später ausführlich und correct in einer Arbeit: "On the electrical response to stimulation of muscle" im Journal of Physiology vol. 18 p. 148 mitgetheilt worden, wo es heisst, dass bei directer Reizung "the electrical change (Schwankung) begins" in "the course of the first thousandth of a Sec. after excitation" und sowohl bei directer als bei indirecter Reizung "marks the invasion of the muscle by the excitatory process"." Mit dem Bedauern, dass das genannte Missverständniss sich in die Mittheilung des Jahres 1890 einschlich, wünscht Sir J. Burdon Sanders on durch die obenstehende briefliche Mittheilung darauf hinzuweisen, dass das Missverständniss bereits in seiner folgenden Mittheilung vollständig berichtigt worden ist.

Wenn also Burdon Sanderson die Zeit für den Eintritt der Negativität der erregten Stelle kürzer als 0,001 Sec. annimmt, so sind auch durch Garten in der allerletzten Zeit abermals werthvolle Beweise für die Hermann'sche Auffassung erbracht worden, dass die Stromesschwankung ohne messbares Intervall nach der Reizung beginnt. Die Versuche am wasserarmen Muskel ergeben nun,

1) Physiolog. Centralbl. 1890 S. 185. 
dass trotz der gewaltigen Steigerung, die das mechanische Latenzstadium erfährt, gar keine Latenz der elektrischen Schwankung aufgetreten ist; ebensowenig hat die Fortplanzung der elektrisehen Erregungswelle irgend eine Aenderung erfahren. Die Versuche ergeben am normalen gerade so wie am wasserarmen Muskel eine Fortpflanzungsgeschwindigkeit, die von 2,78 bis 3,2 Meter pro Sec. als äussersten Grenzen schwankt. Im Mittel berechnete sich aus allen Beobachtungen am normalen Thier $3,15 \mathrm{~m}$, aus jenen mit wasserarmen Muskeln $3,03 \mathrm{~m}$. Unter Zugrundelegung dieser Werthe berechnete sich die Zeit für die gemessene Strecke zwischen Reizelektrode und centraler Ableitungselektrode und dadurch weiter der Moment des Beginns der Schwankung, der dem Reizmoment entsprochen haben würde. Es wurden dabei stets Zahlen erhalten, die um $0,4 \sigma$ von jenen im Maximum abwichen, welche thatsächlich für das Eintreffen der Negativität an der Ableitungselektrode sich aus der Curve durch Messung ablesen liessen, so dass der oben angeführte Satz - es findet sich im wasserarmen Muskel keine nachweisbare Latenz des elektrischen Vorganges - aufgestellt werden konnte.

Aus dem Umstande, dass das mechanische Latenzstadium eine ganz bedeutende Vergrösserung erfährt, während die erste Phase der Actionsströme doch gerade so wie am vormalen Muskel mit dem Reizmomente beginnt, ergibt sich mit einer gewissen Wahrscheinlichkeit der Schluss, dass die graphische Aufzeichnung eines Zeitraumes zwischen Reiz und mechanischer Zustandsänderung auf einer "Latenz der Methodik" und nicht einer Latenz des Muskels beruht. Fast Jahr um Jahr wird durch die Verfeinerung der Technik das mechanische Latenzstadium des Muskels kürzer befunden, so dass es im Laufe von kaum 20 Jahren bereits vier Fünftel seiner Grösse eingebüsst hat, und doch benachrichtigt uns die Methodik noch gar nicht über die Zustandsänderung des Muskelelementes. Vom normalen zum wasserärmsten Muskel, den man von einem lebenden Thier noch gewinnen kann, ist eine ständige Reihe gegeben, in der die Latenz des Muskels langsam zunimmt. Sieht man nun die Curven 5-8 auf dem Curvenblatt genauer an, so findet man wohl ziemlich leicht eine Erklärung hierfür. Wenn auch aus dem annähernd parallelen Verlauf des ansteigenden Schenkels der Curven hervorgeht, dass die Zeit, in der die maximale Verkürzung erreicht wird, ungefähr die nämliche ist, so findet sich doch am Fuss der Curve ein ganz charakteristisches, viel flacher verlaufendes Stückchen, das bei 
Ueber die elektromotorischen Wirkungen des wasserarmen Muskels. 477

Curve 7 schon fast 0,010 Sec. dauert, während es bei geringeren Wasserverlusten auch weniger deutlich ist. In Beobachtungen mit stärkerer Hebelvergrösserung tritt es natürlich bereits auffallend hervor. Dass mit weitergehendem Wasserverlust der ganze ansteigende Schenkel eine wesentlich geringere Steilheit aufweist, wurde bereits oben erwähnt; es ist dies durch den ersten Blick auf Curve 3 und 4 ersichtlich.

Die Verkürzung der Muskelelemente erfolgt also im wasserarmen Muskel im Beginne der Contraction bedeutend langsamer, während sie bei mittleren Wasserverlusten im weiteren Verlauf der Verkürzung noch ziemlich jener am normalen Muskel entspricht. Das, was aber als erste mechanische Lageänderung registrirt werden kann, setzt im Muskel bereits die Entwicklung einer gewissen Energiegrösse, sowie einer bestimmten Dimensionsänderung voraus. Auch wenn man den leichtesten Hebel anwendet oder das leichteste Spiegelchen, muss diese doch zuerst sich entwickelt haben. Mechanisch liegen die Verhältnisse beim suspendirten Muskel ungünstig, indem das Heben des eigenen Gewichts des Muskels, sowie die Dehnung der übrigen Theile des Muskels ( $G$ ad) dazu kommt; dafür ist aber der Weg ein grösserer als bei der Verzeichnung der Dickenzunahme, bei der zwar auch die Veränderung der Widerstände durch die Reibung auf der Unterlage keine zu vernachlässigende ist. Ist nun die Geschwindigkeit, mit der sich das Muskelelement im Beginne nach der Reizung zusammenzieht, wie aus der Curve hervorgeht, eine geringere, somit der Weg, aher auch die erreichte Energiegrösse im Zeittheilchen kleiner, so wird die Latenz um jenen Betrag ansteigen müssen, der dieser Vergrösserung entspricht. Desshalb, weil die graphisch gemessene Latenz gewachsen ist, ist sicher nicht die wirklich gegebene Latenz um denselben Betrag angestiegen. Nun würde einer Zuckungscurve, wie sie dem Curvenblatt (Curve 7) entspricht, nur eine Verlängerung des Latenzstadiums um $3 / 1000-4 / 1000$ Sec. gegen über der Norm zukommen, die Graphik lehrt uns aber, dass dieselbe Verkürzung erst etwa 10/100u Sec. später erreicht ist als am normalen Muskel, wenn man von denselben Fusspunkten auf der Abscissenachse ausgeht; auch die früheren Versuche an arbeitenden wasserarmen Muskeln lassen auf Aehnliches schliessen. Man wird daher nicht fehlgehen, wenn man die Latenzverlängerung des wasserarmen Muskels nur als einen Ausdruck langsamer ansteigender Energie und langsamer sich entwickelnder Verkürzung ansieht, ohne 
dabei anzunehmen, dass das mechanische Geschehen im Muskelelement später einsetzt als am normalen Muskel.

Wenn man bedenkt, dass die Schwierigkeit der graphischen Latenzbestimmungen bereits am normalen Muskel meist an der Bestimmung der Fusspunkte der Curven lag, somit darin schon ein sicheres Zeichen gegeben war, dass unsere direct graphisch oder durch Contactauslösung gewonnenen Latenzwerthe für diesen noch viel zu grosse sein müssen, und die Curve also aussagt, dass die Energieentwicklung anfänglich eine langsame ist, so ist obige Annahme um so wahrscheinlicher. Für den wasserarmen Muskel wird der noch wesentlich flachere Anstieg nur besagen, dass durch die beginnende Schädigung der contractilen Substanz und die grössere Energieentwicklung, welche erforderlich ist wegen der grösseren Widerstände, die bei der tejgigen Beschaffenheit des Muskels gegeben sind, ein grösserer Betrag an innerer Arbeit im Muskel geleistet sein muss, bevor eine mechanische Lageänderung nach aussen wahrnehmbar zum Ausdruck kommen kann.

Findet sich somit kein Grund, das grosse mechanische Latenzstadium, das die Graphik am wasserarmen Muskel ermittelt, auch auf eine längere Latenz im wasserarmen Muskelelement zu beziehen, so ist der Sprung von den zwei Tausendstel-Secunden der mechanischen Latenzzeit des normalen Muskels bis zum vollständigen Fehlen einer solchen für das Maskelelement, das ist zur Identificirung. von Reizmoment, Beginn der Schwankung und Beginn der mechanischen Zustandsänderung, kein grosser und auch kein gewagter mehr. Vermag die Art, in der die Energieentwicklung graphisch zum Ausdruck kommt, durch die Aenderung der Geschwindigkeit, mit der sie im Beginne ansteigt, um das Fünffache zu schwanken, so liegt in der geschlossenen Reihe, welche ein ständiges Sinken dieses Intervalls bis auf jenen Werth zeigt, den man im Muskel des normalen Thieres finden kann, ein Hinweis darauf, wie viel in dem Anstiegstheil der Curve der steigenden Energie bereits im Versuch am normalen Muskel für das Erkennen des Vorganges im Muskelelement sich rein methodisch unserer Beobachtung entzieht. Die Uebereinstimmung der Processe am normalen und wasserarmen Muskel, insbesondere aber das Fehlen einer Latenz in der elektrischen Schwankungscurve des wasserarmen Präparates, berechtigt also wohl im Zusammenhalte mit der Zuckungscurve desselben zur Annahme, dass an diesem ebenso wie eine elektrische Latenz auch ein mecha- 
nisches Latenzstadium des Muskelelementes fehlt, und dass das, was wir als mechanische Latenz des Muskels bezeichnen, zum Theil ein Ausdruck der Latenz der eigenen Methode, zum Theil ein Symbol der Geschwindigkeit der Energieentwicklung ist.

Auch das Feblen einer wesentlichen Aenderung der Leitungsgeschwindigkeit im Nerven bei der Wasserverarmung scheint durch die Vorgänge im Muskel des durstenden Frosches bereits seine Erklärung zu finden. Es bliebe nur noch die Begründung für die wesentliche Verlängerung der "Endorganlatenz" übrig, doch auch diese dürfte nicht schwer fallen.

Wenn in der voranstehenden Mittheilung dargelegt ist, dass trotz dem Sinken des Wassergehaltes eine Aenderung in der Geschwindigkeit des Ablaufes der Schwankungswelle nicht stattfindet, und diese gerade so wie im normalen Muskel mit dem Reizmomente einsetzt; wenn sich ferner ergab, dass im wasserarmen Muskel auf den einzelnen Inductionsschlag periodische Stromesschwankungen auftreten, die denselben Rhythmus aufweisen wie am normalen Muskel bei verschiedenen Formen der Dauercontraction oder bei reflectorischen Erregungen, welche vom Centralnervensystem ausgehen, so ist dadurch wohl auch für die Frage nach der Theorie der Muskelcontraction ein neuer, nicht unwesentlicher Beitrag erbracht. Denkt man nun an das Wandern von Wassermolekülen, an ganz bestimmte Aenderungen der Oberflächenspannung oder an Aenderungen der Ionenbeweglichkeit, immer werden sich die am wasserarmen Muskel gefundenen Thatsachen in die Theorie fügen müssen, soll diese Anspruch auf Richtigkeit haben. 